\title{
Truth as a Mathematical ObJect
}

\author{
JEAN-Yves BÉZIAU \\ Federal University of Fortaleza/CNPq
}

\begin{abstract}
In this paper we discuss in which sense truth is considered as a mathematical object in propositional logic. After clarifying how this concept is used in classical logic, through the notions of truth-table, truth-function and bivaluation, we examine some generalizations of it in non-classical logics: many-valued matrix semantics with three and four values, non-truth-functional bivalent semantics, Kripke possible world semantics.
\end{abstract}

Keywords: Truth, truth-table, truth-function, bivaluation, many-valued logic, paraconsistent logic, possible worlds.

Dedicated to Newton da Costa for his 80th birthday

Si les mathématiques n'étaient qu'un jeu je me serais contenté de jouer à la poupée

BARON DE CHAMBOURCY

\section{Introduction}

A fundamental feature of modern logic by opposition to traditional logic (e.g. Aristotelian logic) is the use of mathematics as a tool, like in many other sciences. In the XIXth century people such as Boole and Schröder have contributed to use arithmetic and algebra to deal with reasoning.

How can we consider truth as a mathematical object? Many people when they hear about truth in the context of modern logic immediately think of Tarski's theory of truth. But beside and before this theory, truth was considered as a mathematical object in propositional logic, through the notion of truth-value.

It seems that Heinrich Windelband was the first to use the expression "truthvalue" in 1884 (see Gabriel 1986). According to Windelband, truth-value means that in some sense truth is valuable and this is certainly true. But it is also true that it may be valuable to consider truth as a value in a mathematical sense, in a functional sense: truth as the value of a function. ${ }^{1}$ In this mathematical sense, not only truth is valuable, but also falsity is: truth and falsity are both values, both are considered as "truth-values". But is falsity the only other truth-value? Can we consider for example with Łukasiewicz that possibility is a truth-value?

From a mathematical point of view it is easy to multiply the truth-values, but does this make sense? Two people have reacted against this: Quine saying that Principia 14(1): 31-46 (2010).

Published by NEL — Epistemology and Logic Research Group, Federal University of Santa Catarina (UFSC), Brazil. 
many-valued logic is just algebra (Quine 1970) and Suszko in a subtler way making the distinction between logical values and algebraic values (Suszko 1977).

We are facing here a key problem of the connection between mathematics and philosophy. Mathematics can be a useful tool to go further in the analysis of some philosophical ideas. Mathematics is a powerful tool, but we must be cautious with such kind of power, avoiding to develop some abstract mental constructions which have nothing to do with the initial philosophical problems we wanted to deal with see the famous paper by Gian-Carlo Rota: "The pernicious influence of mathematics upon philosophy" (Rota 1991).

In this paper we will describe the main mathematical tools used to deal with truth in propositional logic and discuss the philosophical perspectives related to them.

\section{Truth in classical propositional logic}

\subsection{Truth-tables}

A central feature of the semantics of classical propositional logic is the notion of truth-table. Truth-tables can be presented in different ways. One of the most common presentations of a truth-table is derived from the Tractatus of Wittgenstein (4.31), it is like this:

\section{Wittgensteinian truth-table}

\begin{tabular}{|c|c|c|}
\hline$p$ & $q$ & $p \wedge q$ \\
\hline $\mathrm{F}$ & $\mathrm{F}$ & $\mathrm{F}$ \\
\hline $\mathrm{F}$ & $\mathrm{T}$ & $\mathrm{F}$ \\
\hline $\mathrm{T}$ & $\mathrm{F}$ & $\mathrm{F}$ \\
\hline $\mathrm{T}$ & $\mathrm{T}$ & $\mathrm{T}$ \\
\hline
\end{tabular}

In a Wittgensteinian truth-table $\mathrm{F}$ and $\mathrm{T}$ are abbreviations for Falsity and Truth. A Wittgensteinian truth-table can be described in natural language saying

the conjunction of two propositions is true if and only if both are true.

Why using a truth-table rather than a sentence of natural language? By using a truthtable are we doing a kind of mathematics of truth-values? Tables have connections with mathematics; for example children learn addition and multiplication through some tables. But it is important to note that tables are used also outside mathematics, to organize thought in a clearer way, as done with all kinds of diagrams. This is what we can call pictorial thinking (the second Wittgenstein was keen of pictorial thinking). To put truth in a table can mean two different (non-excluding) things: 
- to mathematize truth in a computational sense

- to picture truth.

The formulation in the table is more precise because we see that a proposition cannot be both true and false and that it has to be either true or false (principle of bivalence). ${ }^{2}$ In the natural language formulation this has to be added: the above formulation in natural language holds for some semantics which do not obey the principle of bivalence.

Instead of using $\mathrm{F}$ and $\mathrm{T}$ in a truth-table, it is possible to use 0 and 1 :

0-1 truth-table

\begin{tabular}{|c|c|c|}
\hline$p$ & $q$ & $p \wedge q$ \\
\hline 0 & 0 & 0 \\
\hline 0 & 1 & 0 \\
\hline 1 & 0 & 0 \\
\hline 1 & 1 & 1 \\
\hline
\end{tabular}

What is the difference and what is the importance of doing that? Are we getting more mathematical? It is possible to represent falsity and truth by any kind of signs. 0 and 1 have the power of abbreviation, but so have $\mathrm{F}$ and T. Are 0 and 1 more symbolic than F and T? Maybe: falsity is connected with the emptiness of the zero and truth with the strength of the firstness of the one. But there is more than this; we can use the mathematical properties of 0 and 1 . For example we can define conjunction taking in account the order relation between 0 and 1, using the notion of inf.

What is the connection between a truth-table and a table for addition or multiplication? If we want to represent a truth-table like a multiplication table it will look like this:

\section{Proper truth-table}

\begin{tabular}{|l|l|l|}
\hline$\wedge$ & 0 & 1 \\
\hline 0 & 0 & 0 \\
\hline 1 & 0 & 1 \\
\hline
\end{tabular}

Note then that $p, q, p \wedge q$ have disappeared. This is because conjunction is a function whose domain is $\{0,1\}$ and whose co-domain is also $\{0,1\}$. This table can be replaced by the following equations

$$
\begin{aligned}
& 0 \wedge 0=0 \\
& 0 \wedge 1=0 \\
& 1 \wedge 0=0 \\
& 1 \wedge 1=1
\end{aligned}
$$

Principia 14(1): 31-46 (2010). 
In this case we have a kind of arithmetization of truth, we could use the symbol $\times$ or the symbol - instead of $\wedge$. It has been done and also the following kind of equation has been written:

$$
p \cdot p=p
$$

This has been done in the Boolean tradition. But is conjunction an operation on propositions or numbers? This is not clear. In the Boolean tradition one can find equations where there is a mix of numbers and propositions such as

$$
p \cdot \neg p=0
$$

And when we have an equation expressing the commutativity of the conjunction

$$
p \wedge q=q \wedge p
$$

we may wonder if $p$ and $q$ are variables ranging over a domain like when we write

$$
x \times y=y \times x,
$$

where variables are ranging over numbers. In the case of propositional logic $p$ and $q$ can be viewed as variables ranging over 0 and 1 . But they can also be viewed as variables ranging over propositions: atomic formulas in propositional logic are called propositional variables. But the above equation holds for any formulas, so one could say that $p$ and $q$ are formula variables.

\subsection{Truth-functions}

Something that does not appear in most of logic books is that the semantics of classical propositional logic (hereafter CPL) is the set of homomorphisms between the absolutely free algebra of the set of formulas and the Boolean algebra on $\{0,1\}$. Homomorphism means that we have two structures of the same type, i.e. with the same kind of operators. On the one hand we have the connectives, on the other hand something we can call truth-functions, functions which have as domain and co-domain the set $\{0,1\}$ and that can be defined via proper truth-tables, as the one we have presented in the previous section for conjunction.

This picture is not clear for many people for various reasons:

(a) The set of formulas is not generally presented as an algebra, it is presented in a rather linguistic way with words like: "alphabet", "language", etc. To consider the set of formulas as an absolutely free algebra is meaningless for most people, except for those working in algebraic logic (see Beziau 1999c).

(b) People use the same notation for the connectives and the corresponding truthfunctions - in the case of conjunction for example the symbol $\wedge$. 
(c) When talking about Boolean algebra in the context of CPL, many people are thinking of a Boolean algebra not as a logical matrix but as the factor structure we get using the notion of logical equivalence - a fact that has nothing to do with truth-value: all logics in which logical equivalence is a congruency can be factorized in this way even if they don't have a semantics of truth-values in the sense of a finite characteristic matrix, this is the case for example of intuitionistic logic or the modal logic S5.

(d) There is often a confusion between bivaluations and truth-functions: bivaluations are sometimes called truth-functions.

E. Post (1921) has shown that CPL is truth-functionally complete. One may think that this property is a good reason to defend CPL. But other logics are truthfunctionally complete and this is only one aspect of the strength of a logic: Post has also shown that CPL is maximal (but other logics also are maximal).

What is truth-functional completeness? It means that from the truth-functions of CPL it is possible to define all truth-functions of finite arity: all finitary functions on $\{0,1\}$. As it is known we don't need all the basic truth-functions of conjunction, implication, negation, etc. One may be enough, like Sheffer stroke.

Making the distinction between truth-functions and connectives is important to understand the limitation of Post maximal theorem. This theorem says that the connectives of classical logic cannot be strengthened. But it does not mean that all possible connectives can be defined using connectives of CPL. For example the modal operator $\diamond$ cannot be defined in CPL.

We have to remember also that it is possible to have a logic weaker than classical logic defined by truth-functions - for example positive classical logic defined by the truth-functions of conjunction, disjunction and implication - but that some logics weaker than classical logic, e.g. intuitionistic logic, cannot be defined by truthfunctions.

\subsection{Bivaluations}

The bivaluations of the semantics for CPL have to be clearly distinguished from the truth-functions. They are the homomorphisms between the structure of the formulas and the structure of truth-values.

Bivaluations can reduce to something which is sometimes called distributions of truth-values. These are functions whose domain is the set of atomic formulas and whose co-domain is $\{0,1\}$. One can prove that for each distribution of truth-values there is a unique extension of it whose domain is the set of all formulas and whose co-domain is $\{0,1\}$ and that any bivaluation can be generated by a distribution of truth-values. The concept of distribution of truth-values is related to the concept of 
atomic formula, which makes sense through the notion of absolutely free algebra. And in fact the above theorem touches a fundamental property of an absolutely free algebra, see (Beziau 1999c).

How is truth distributed? Why such and such atomic proposition should be true in a given distribution? In the Tractarian perspective this has no value. One considers only all possible distributions and then focus on what holds for all bivaluations (extensions of distributions): the tautologies, as Wittgenstein used to say, which are also called logical truths. Unlike truth, logical truth is not considered as a number: 0,1 or 9.

What we have here is a theory of logical truth rather than a theory of truth. Why an atomic proposition like "Snow is black" is true or not, this is never discussed. The logician will be happy enough to say that "Snow is black or snow is not black" is logically true. As pointed out by Wittgenstein, then we are saying nothing about the world (see e.g. Tractatus 4.461).

To deal with truth we can go in two different directions: first-order logic where atomic propositions are atomized - at this level Tarski has developed his theory of truth which led to model theory - or a theory of truth related to the way the brain works (see Suppes \& Beziau 2004).

There is another way to present this same set of bivaluations, i.e the semantics of CPL. We can see bivaluations as characteristic functions of maximal consistent sets of formulas. But what is a maximal consistent set of formulas? This notion is beyond the distinction between semantics and proof-systems ${ }^{3}$ and also is abstract in a sense that it does not depend on a specific language or some specific connectives. Given a relation of consequence, we say that a set is maximally consistent iff:

(a) not everything is a consequence of it,

(b) any strict extension of it is inconsistent (everything is a consequence of it) .

We can define the notion of maximal consistent set on the basis of a consequence relation proof-theoretically defined or semantically defined.

In CPL it is possible to prove that bivaluations are exactly characteristic functions of maximal consistent sets of formulas semantically defined and also prooftheoretically defined. The concept of maximal consistent set of formulas is a key notion for the completeness theorem.

The fact that in CPL bivaluations are at the same time homomorphims and maximal consistent sets is a striking feature of CPL semantics. The two different ways to consider bivaluations lead to two generalizations of the semantics of CPL in which this coincidence does not necessarily hold.

Principia 14(1): 31-46 (2010). 


\section{Truth in many-valued logic}

\subsection{Logical matrix}

A straightforward generalization of classical propositional bivalent semantics is many-valued matrix semantics, generally just called many-valued semantics.

The idea is to consider homomorphisms between a structure of formulas and a structure of similar type, called a logical matrix, with a domain of a given cardinality divided in two sets: the set of designated values and the set of non-designated values. This division is used to define the set of tautologies and the consequence relation. The cardinality of the domain of the matrix is supposed to be strictly superior to 1 . It can be finite or not.

Lindenbaum has shown that any logic can be characterized by a matrix; this is due to the structural character of a logic, i.e. the fact that the set of tautology and the consequence operator are invariant under substitutions. For this purpose Lindenbaum used substitutions as truth-functions. ${ }^{4}$

Despite this fact, generally people will not say that a logic is many-valued because it can be characterized by a logical matrix, they will use this expression only to qualify logics that can be characterized by a finite matrix (whose domain cardinality is superior to 2). For example the modal logic S5 which cannot be characterized by a finite matrix due to a famous result of Dugundji (1940) is generally not known as a many-valued logic, the same with intuitionistis logic (see Beziau 1997). And $\mathrm{CPL}$ is not considered as many-valued -2 is not so many.

\subsection{Eukasiewicz three valued logic and the meaning of the third value}

A simple example of logical matrix different from the bivalent one of CPL is the one for Łukasiewicz three-valued logic L3. In this logic the truth-table for negation is the following:

Łukasiewicz truth-table for negation

\begin{tabular}{|c|c|}
\hline & $\neg$ \\
\hline $\mathrm{F}$ & $\mathrm{T}$ \\
\hline $\mathrm{I}$ & $\mathrm{I}$ \\
\hline $\mathrm{T}$ & $\mathrm{F}$ \\
\hline
\end{tabular}

$\neg$ is a function whose domain and co-domain is the three-valued set $\{F, I, T\}$. I is an abbreviation for indeterminate. It is common to say nowadays that indeterminate is a third value. But is it really a truth-value? And can we say that $\neg$ here is a truth-function? 
The motivation of Łukasiewicz to consider a third value was related to determinism. According to Łukasiewicz, if we say that the proposition "It will rain tomorrow" is either true or false, then we are committed to determinism. To avoid determinism we can say that the truth-value of this proposition is indeterminate. But in a truthtable this indetermination turns into an object which stands at the same level as truth and falsity. Maybe formalization leads here to ambiguity. Łukasiewicz alternatively considered the third value as possible. It is perhaps less ambiguous to consider that possibility is a truth-value than to consider indetermination of truth-value as a truth-value.

In both cases the third value is considered as non-designated. One may wonder if it would not be better to call truth any designated value and falsity any nondesignated values, to therefore avoid using these names for the values within the tables, i.e. as names for the objects of the domain of the matrix. This was the idea of Suszko who suggested to call these objects, algebraic values and not truth-values, to avoid the madness of the multiplication of truth-values ("any multiplication of logical values is a mad idea", Suszko 1977).

If we use truth and falsity for values of the matrix we are led to some incoherencies: for example $\neg$ of $Ł 3$ is a contradictory forming functor since $p$ is true if and only if $\neg p$ is false, but at the same time it has not the property of classical negation, for example $\neg(p \wedge \neg p)$ is not a tautology. Contrarily it seems good to use truth for designated values and falsity for non-designated values, because then we can say that logical consequence is truth preserving: it leads from truths to truths.

But one may also have another conception of logical consequence according to which it is a path from some hypotheses to a conclusion and argue that it is also possible to coherently speak about truth and falsity within truth-tables of manyvalued logics, as we will see.

Using the same truth-table as the one for $\neg$ in $Ł 3$, it is possible to change the set of designated and non-designated values considering that $\mathrm{F}$ is non-designated and that I and $\mathrm{T}$ are designated. This is the idea of paraconsistent logicians: Asenjo (1966), D'Ottaviano and da Costa (1970) and Priest (1979). In this case from $p$ and $\neg p$ it is not possible to deduce anything. Priest has tried in this context to give an intuitive justification of the third truth-value, saying that a proposition can be truefalse or true and false (Priest 1979). The second way of speaking can be emphasized through the following truth-table:

Priest truth-table for negation

\begin{tabular}{|c|c|}
\hline & $\neg$ \\
\hline$\{\mathrm{F}\}$ & $\{\mathrm{T}\}$ \\
\hline$\{\mathrm{T}, \mathrm{F}\}$ & $\{\mathrm{T}, \mathrm{F}\}$ \\
\hline$\{\mathrm{T}\}$ & $\{\mathrm{F}\}$ \\
\hline
\end{tabular}

Principia 14(1): 31-46 (2010). 
Formally speaking it is quite the same mathematical structure. The three objects have just three different names. But such an approach would be mathematically nicer if we had all the elements of the power set of $\{\mathrm{F}, \mathrm{T}\}$ - here we don't have the empty set.

Anyway Priest's interpretation makes sense considering a proposition like "It is raining now in Brazil". If it is raining in Rio de Janeiro but not in Brasilia, this proposition can be considered as true and false, its negation can also be considered as true and false and from this proposition and its negation, we don't have good reason to deduce everything.

We can also consider the three values as numbers:

\begin{tabular}{|c|c|}
\hline & $\neg$ \\
\hline 0 & 1 \\
\hline$\frac{1}{2}$ & $\frac{1}{2}$ \\
\hline 1 & 0 \\
\hline
\end{tabular}

Using numbers gives the possibility to use the linear order between them to define conjunction and disjunction as inf and sup, whether we are considering $\frac{1}{2}$ as designated or not. It is also possible to consider non-linear orders. This is typically the case of Dunn-Belnap four-valued semantics we will study next.

\subsection{Dunn-Belnap four valued logic: four truth-values making sense}

The idea of Dunn-Belnap semantics (see e.g. Belnap 1977) is to use a structure on truth-values which is a very simple de Morgan lattice. A good example of such a lattice is given by the power set of a two elements sets whose elements are ordered by the inclusion relation. For truth-values we can of course take as a two elements set, the set with truth and falsity. In this case it does not matter if we use 0 and 1 rather than $\mathrm{F}$ and $\mathrm{T}$ to represent these objects, since we will not use properties of these names for the definition of truth-tables. Here is a way to represent the truth-table for negation in Dunn-Belnap semantics:

Dunn-Belnap truth-table for negation

\begin{tabular}{|c|c|}
\hline & $\neg$ \\
\hline$\{\mathrm{F}\}$ & $\{\mathrm{T}\}$ \\
\hline$\emptyset$ & $\emptyset$ \\
\hline$\{\mathrm{T}, \mathrm{F}\}$ & $\{\mathrm{T}, \mathrm{F}\}$ \\
\hline$\{\mathrm{T}\}$ & $\{\mathrm{F}\}$ \\
\hline
\end{tabular}

If we consider $\{F\}$ and $\emptyset$ as non-designated and $\{T\}$ and $\{T, F\}$ as designated we have something that is called a de Morgan logic. $\{\mathrm{T}, \mathrm{F}\}$ can be interpreted as truefalse and $\emptyset$ as nonsense or indetermination. It means that the proposition has no 
truth-value, it is neither false, nor true. We can think this is the case of propositions like "It will rain tomorrow" or "Four is red".

The Dunn-Belnap semantics looks very nice and it seems not condemnable by Suszko. There is no multiplication of truth-values but what we have is the mathematical strength of truth and falsity via the power set operation and the non-linear order naturally given by the inclusion relation.

Shramko and Wansing (2005) have argued that there is also a very coherent interpretation of 16 truth-values based on a four elements set, using in the same way the power set operation and the order relation generated via inclusion. They call generalized truth-values these structures of truth-values generated by the power set operation and the relation of inclusion.

Now what about pure multiplication of truth-values, e.g. according to an idea of degrees of truth and falsity? One may consider the set of real numbers (this is not so clever because this is a linear order) and define conjunction as inf, disjunction as sup, and negation by taking the inverse. Then one says that the positive real numbers are designated values and the negative ones, non-designated ones. It happens that doing this, one is defining exactly classical logic. This is where the multiplication of truth-values seems absurd because the distinction between designated and nondesignated value to define the set of tautologies and the consequence relation leads us back to bivalence and produces the collapse.

If one really wants to consider degrees of truth, one should perhaps also consider degrees of tautology and degrees of consequence using some functions reflecting the degrees of truth of truth-functions on tautologies and consequences. Then one would be able to say that there are tautologies which are for example $\pi$ logically true.

\section{Truth in non-truth-functional bivalent semantics}

\subsection{Suszko bivalent semantics for $Ł 3$}

Suszko was against more truth-values than truth and falsity and showed how to construct a bivalent semantics for Łukasiewicz logic Ł3. At first this seems absurd because the reason why $Ł 3$ is considered strictly speaking a three-valued logic is because it cannot be characterized by a two-valued matrix semantics. CPL can be characterized by a three-valued semantics or by a matrix of any cardinality superior to two. When we say that a logic is $n$-valued, it means that $n$ is the smallest cardinal of the set of values of a matrix that characterizes the logic.

The bivalent semantics of Suszko for $Ł 3$ is not a truth-functional bivalent semantics, it is not the set of homomorphisms between an algebra of formulas and an algebra of similar type on $\{0,1\}$. It is the set of characteristic functions of maximal consistent sets of formulas.

Principia 14(1): 31-46 (2010). 
It is possible to understand the fact that any many-valued logic can have a bivalent semantics through the fact that, as Suszko pointed out, bivalency in manyvalued semantics is still there through the distinction between designated and nondesignated value, which is used to define the notion of tautology and the notion of consequence relation.

It is possible to prove some general results showing that most logics can be characterized by bivalent semantics (da Costa et al. 1996). But many-valued logic is not necessarily useless, the reduction to bivalency has its advantages and its disadvantages. It is like many examples of reduction. For example we know that all connectives of classical logic can be reduced to Sheffer stroke, it does not mean that it makes no sense to explicitly use disjunction, conjunction, implication and negation. Also we know that all signs of a language can be reduced to two signs, that can be expressed as 0-1. But it is not necessarily bad to use an alphabet of 26 letters.

What is interesting is to use the reduction when it is useful and to keep the variety when it is better. It is worth also to study the interplay between the two sides. João Marcos has developed a work in this direction, see e.g. Caleiro et al. 2007. Beziau (1999b), using a general theorem connecting bivaluations and sequent rules (Beziau 2001), was able to provide a sequent system for $Ł 3$.

\subsection{Da Costa's theory of valuation}

Da Costa has developed a theory whose aim is to show that any logic can be considered as bivalent, the theory of valuation - which would be better named "theory of bivaluation", see (da Costa \& Béziau 1994; Beziau 2011). His work on the subject started with the study of a particular system of logic: his paraconsistent system C1. He developed a bivalent semantics for this system that can be described using truth-tables (da Costa \& Alves 1977). For example the truth-table for negation is the following:

da Costa truth-table for negation

\begin{tabular}{|c|c|}
\hline$p$ & $\neg p$ \\
\hline 0 & 1 \\
\hline 1 & 0 \\
\hline 1 & 1 \\
\hline
\end{tabular}

What is important here is that this is in no sense a truth-table for $\neg$ as a truthfunction, it is a truth-table for $\neg$ as a connective. For this logic there are no truthfunctions corresponding to the connective $\neg$. Another point is that according to this semantics, as this truth-table clearly shows, when $p$ is true, $\neg p$ can be true or $\neg p$ can be false. How to qualify this behavior? We can say that this is a non-deterministic 
semantics. But we have to be careful because non-deterministic does not mean undecidable. This terminology has been used by Walter Carnielli and Arnon Avron to name semantics which are generalizations of da Costa's one, see (Carnielli 2000; Avron 2007).

Instead of saying that $p$ is true-false or that $p$ is both true and false, we say that $p$ is true and $\neg p$ is true. There is here an interplay between negation, truth and falsity without multiplication of truth-values.

To be true means to be part of a maximal consistent set of formulas. The notion of truth behind this notion is much connected with a vision of truth as coherence and/or completeness. This concept is also fundamentally structuralist, it means that truth of a proposition depends on the relation with other propositions. "It is raining now in Brazil" can be true at the same time that "it is not raining now in Brazil" in the sense that from these two propositions we cannot deduce everything. The quality of this kind of truth is related with what kind of other propositions can also be true with these two, and what we can deduce or not from them.

Bivalent non-truth-functional semantics gives more freedom to develop new theories about connectives and also permits to deal with languages which are not based on the atomic conception of the language. In a bivalent non-truth-functional semantics like the one for C1, bivaluations are defined directly on the set of all formulas and cannot be defined starting with distributions of truth-values on atomic formulas. Truth is global.

\section{Truth in Kripke possible world semantics}

An alternative to many-valued semantics and non-truth-functional bivalent semantics is Kripke possible world semantics. Kripke semantics is a different way to speak about truth, where truth in a world depends on truth in other worlds. This can be used to deal with all kinds of modal operators and also with negation.

For example we can deal with paraconsistent negation in the following way: If $p$ is true in the world $w$ and in all worlds accessible to this world $w$, then $\neg p$ is false in this world $w$. But if $p$ is true in the world $w$ and there is a world accessible from the world $w$ in which $p$ is false, then $\neg p$ is true in this world $w$.

This can be seen as collective thinking, taking in account the opinions of others. If John Smith is a member of a group of people, he may himself think that "it is now raining in Brazil" is true but if there is someone else in this group who thinks that "it is now raining in Brazil" is false, he will think that "It is not now raining in Brazil" is also true. This is connected with Ja?kowski's idea of discussive logic (Beziau 2006a) and it has also been shown that the negation so defined corresponds to "not necessary" (see Beziau 2002, 2005).

Principia 14(1): 31-46 (2010). 
Like in non-truth-functional bivalent semantics true-false is not used, the third value is simulated by an interplay between negation, truth and falsity, an interplay which is more subtle, since we use the accessibility relation and double recurrence to define truth. These are the main characteristics of possible world semantics and not the notion of possible world itself that can be considered as something similar to maximal consistent sets of formulas (see Beziau 2006b).

Possible world semantics maybe is more interesting to deal with negation or implication than with modal operators, because in standard modal logics like S5, we learn quite nothing: a proposition is necessarily true iff it is true in all worlds, so necessity in this case is just the internalization of a meta-property and we have something quite tautological: $\square F$ is a tautology iff $F$ is a tautology. We can understand why Wittgenstein was saying that modalities could not be considered as logical operators, but were metalogical notions (see e.g. Tractatus 5.525). ${ }^{5}$

It is clearly possible to mix many-valued semantics with Kripke semantics considering for example worlds where a proposition can be true, false or true-false. Such constructions may be used for example to combine a paraconsistent logic with a modal logic but the relevance of this kind of construction has still to be inquired (see Beziau 2006b). This is a construction naturally provided by mathematics but whose philosophical counterpart is perhaps dubious or can be expressed in a better way by other mathematical tools. ${ }^{6}$

\section{References}

Asenjo, F. 1966. A calculus of antinomies. Notre Dame Journal of Formal Logic 7: 103-5.

Avron, A. 2007. Non-deterministic matrices and modular semantics of rules. In J.-Y.Beziau (ed.) Logica Universalis - Towards a general theory of logic. 2nd ed. Basel: Birkhäuser, pp. 155-74.

Belnap, N. D. 1977. A useful four-valued logic. In M. Dunn (ed.) Modern uses of multiplevalued logic. Boston: Reidel, pp. 8-37.

Béziau, J.-Y. 1997. What is many-valued logic? In Proceedings of the 27th International Symposium on Multiple-Valued Logic, IEEE Computer Society, Los Alamitos, pp. 117-21.

- 1999a. Classical negation can be expressed by one of its halves. Logic Journal of the Interest Group in Pure and Applied Logics 7: 145-51.

- 1999b. A sequent calculus for Łukasiewicz's three-valued logic based on Suszko's bivalent semantics. Bulletin of the Section of Logic 28: 89-97.

- 1999c. The mathematical structure of logical syntax. In W. A. Carnielli et al. (eds), Advances in contemporary logic and computer science. Providence: American Mathematical Society, pp. 3-15.

- 2001. Sequents and bivaluations. Logique et Analyse 44: 373-94.

. 2002. S5 is a paraconsistent logic and so is first-order logic. Logical Investigations 9: 301-9.

Principia 14(1): 31-46 (2010). 
2003. Bivalence, exluded middle and non contradiction. In L. Behounek (ed. )The Logica Yearbook 2003. Prague: Academy of Sciences, pp. 73-84.

- 2004. Non truth-functional many-valued semantics. In J.-Y. Béziau, A. Costa-Leite and A. Facchini (eds.) Aspects of Universal Logic. Neuchâtel: Université de Neuchâtel, pp. $199-218$

- 2005. Paraconsistent logic from a modal viewpoint. Journal of Applied Logic 3: 7-14. . 2006a. The Paraconsistent logic z - a possible solution to Jaskowski's problem! Logic and Logical Philosophy 15: 99-111.

- 2006b. Many-valued and Kripke semantics. In J. van Benthem et al. (eds.) The age of alternative logics. Dordrecht: Springer, pp. 89-101.

. 2009. Bivalent semantics for De Morgan logic (the uselessness of four-valuedness). In W. A .Carnielli, M. E. Coniglio and I. M. L. D'Ottaviano (eds.) The many sides of logic. London: King's College, pp. 391- 402.

- 2010. What is a possible world? In G. Imaguire and D. Jacquette (eds.) Possible worlds: logic, semantics and ontology. Munich: Philosophia Verlag, pp. 25-37.

- 2011. Paranormal logics and the theory of bivaluations. In J.-Y.Beziau (ed.) Anthology of Universal Logic. Basel: Springer, pp. 221-36.

Caleiro, C.; Carnielli, W.; Coniglio, M.; Marcos, J. 2007. The Humbug of many logical values. In J.-Y. Beziau (ed.) Logica Universalis - Towards a general theory of logic. 2nd ed. Basel, pp. 175-94

Carnielli, W. A. 2000. Possible-translation semantics for paraconsistent logics. In D. Batens et al. (eds) Frontiers of paraconsistent logic. Baldock: Research Studies Press, pp. 149-63.

da Costa, N. C. A. \& Alves, E.H. 1977. A semantical analysis of the calculi Cn. Notre Dame Journal of Formal Logic 16: 621-30.

da Costa, N. C. A. \& Béziau, J.-Y. 1994. Théorie de la valuation. Logique et Analyse 146: 95-117.

da Costa, N. C. A.; Béziau, J.-Y.; Bueno, O. A. S. 1996. Malinowski and Suszko on manyvalued logics: On the reduction of many-valuedness to two-valuedness. Modern Logic 6: 272-99.

D’Ottaviano, I. M. L. \& da Costa, N. C. A. 1970. Sur un problème de Jaskowski. Comptes Rendus de l'Académie des Sciences de Paris 270: 1349-53.

Dugundji, J. 1940. Note on a property of matrices for Lewis and Langford's calculi of propositions. Journal of Symbolic Logic 5: 150-1.

Gabriel, G. 1984. Fregean connection: Bedeutung, Value and Truth-Value. The Philosophical Quarterly 34: 372-6.

- 1986. Kant als Neukantianer. Kant Studien 77: 84-101.

Kripke, S. 1959. A completeness theorem in modal logic. Journal of Symbolic Logic 24: 1-14.

Łoś, J. 1949. O matrycach logiczych. Travaux de la Société des Sciences et des Lettres de Wroclaw, série B, 19.

Łukasiewicz, J. 1920. O logice trojwartosciowej. Ruch Filozoficny 5: 170-1.

Post, E. 1921. Introduction to a general theory of propositions. American Journal of Mathematics 43: 163-85.

Priest, G. 1979. The logic of paradox. Journal of Philosophical Logic 8: 219-41.

Quine, W. V. O. 1970. Philosophy of logic. 2nd ed. Cambridge, Mass.: Harvard University Press, 1986.

Principia 14(1): 31-46 (2010). 
Rota, G. C. 1991. The pernicious influence of mathematics upon philosophy. Synthese 88: 165-78.

Shramko, Y. \& Wansing, H. 2005. Some useful 16-valued logics: how a computer network should work. Journal of Philosophical Logic 34: 121-53.

Suppes, P. \& Béziau, J.-Y. 2004. Semantic computation of truth based on associations already learned. Journal of Applied Logic 2: 457-67.

Suszko, R. 1977. The Fregean axiom and Polish mathematical logic in the 1920s. Studia Logica 36: 87-90.

Wittgenstein, L. 1921. Tractatus Logico-Philosophicus. Annalen der Naturphilosophie 14: 185362.

Wojcicki, R. 1969. Logical matrices strongly adequate for structural sentential calculi. Bulletin de l'Académie Polonaise des Sciences - Série des Sciences Mathématiques, Astronomiques et Physiques 17: 333-5.

JEAN-Yves BÉZIAU

Departmento de Filosofia

Universidade Federal do Rio de Janeiro Largo de São Francisco de Paula, 1 20051-070 Rio de Janeiro, RJ BRAZIL

jyb@ufc.br

Resumo. Neste artigo, discutimos em que sentido a verdade é considerada como um objeto matemático na lógica proposicional. Depois de esclarecer como este conceito é usado na lógica clássica, através das noções de tabela de verdade, de função de verdade, de bivaloração, examinamos algumas generalizações desse conceito nas lógicas não clássicas: semânticas matriciais multi-valoradas com três ou quatro valores, semântica bivalente não veritativa, semânticas dos mundos possiveis de Kripke.

Palavras-chave: Verdade, tabela de verdade, função de verdade, bivaloração, lógica polivalente, lógica paraconsistente, mundos possíveis.

\section{Notes}

${ }^{1}$ Who was the first to consider truth-value as a value of a function, we don't know exactly - the aim of this paper is not historical. To say, like Frege, that truth-value is the reference (Bedeutung) of a proposition does not necessarily imply that it is considered as a value of a function, unless a proposition is considered as a function (like in Wittgenstein's Tractatus, 5: "A proposition is a truth-function of elementary propositions"). About Frege, see (Gabriel 1984).

${ }^{2}$ About the exact formulation of the principle of bivalence, its relation with truth, falsity and negation, see (Beziau 2003).

${ }^{3}$ We are avoiding here to use the word "syntax" because it can lead to a confusion between proof-theory and the construction of the set of formulas; these are two different things.

Principia 14(1): 31-46 (2010). 
${ }^{4}$ Lindenbaum theorem was first published by (Łoś 1949), it was for logics conceived as sets of formulas, it was then generalized to structural consequence operators by Łoś, Suszko and Wójcicki (see Wójcicki 1969). In view of Lindenbaum theorem we can understand the point 5.01 of Wittgenstein's Tractatus: "Elementary propositions are the truth-arguments of propositions."

${ }^{5}$ Wittgenstein was not using the terminology "metalogical", but this is how we can interpret nowadays what he was saying.

${ }^{6}$ Member of the LogComp project (CNPq-universal). Work supported by a grant DCR of FUNCAP/CNPq. Thank you to Tarcisio Pequeno, Guido Imaguire and Fabien Schang.

Principia 14(1): 31-46 (2010). 